\title{
Insegurança alimentar em agricultores: violação do direito humano à alimentação adequada
}

\section{Food insecurity in farmers: violation of the human right to adequate food}

Lucas Alves da Costa' , Amanda de Andrade Marques' (D), Juliana Ribeiro Francelino Sampaio ${ }^{2}$ (D), Willma José de Santana ${ }^{3}$ (D) Elida Mara Braga Rocha ${ }^{1 *}$ (D)

${ }^{1}$ Centro Universitário de Juazeiro do Norte (UNIJUAZEIRO) - Juazeiro do Norte (CE), Brasil.

${ }^{2}$ Faculdade Estácio de Medicina de Juazeiro do Norte (FMJ) - Juazeiro do Norte (CE), Brasil.

${ }^{3}$ Faculdade de Tecnologia (FATECICENTEC - CARIRI) - Juazeiro do Norte (CE), Brasil.

Como citar: Costa LA, Marques AA, Sampaio JRF, Santana WJ, Rocha EMB. Insegurança alimentar em agricultores: violação do direito humano à alimentação adequada. Cad Saúde Colet, 2021;29(1):122-132. https://doi.org/10.1590/1414$462 \times 202129010471$

\section{Resumo}

Introdução: No Brasil, vislumbraram-se avanços significativos nas políticas de alimentação e nutrição. Apesar disso, o país apresenta milhões de pessoas em condições de Insegurança Alimentar (IA), com reconhecida dificuldade de acesso à alimentação adequada. Objetivo: Esta pesquisa teve como objetivo investigar a associação entre insegurança alimentar com o perfil antropométrico e socioeconômico de agricultores dos municípios da região centro-sul do Ceará. Método: Trata-se de uma pesquisa transversal que utilizou um questionário socioeconômico e a Escala Brasileira de Insegurança Alimentar (EBIA) para avaliar a situação de IA e o Índice de Massa Corporal (IMC) para avaliação do estado nutricional. Os testes de Mann-Whitney e qui-quadrado foram utilizados, considerando significativo $p<0,05$. Resultados: Participaram desta pesquisa 86 agricultores, destes 39,5\% em condições de IA e 41\% com sobrepeso. Foi identificada associação entre a situação de IA em famílias com o maior número de indivíduos $(p<0,05)$, a presença de menores de 18 anos $(p<0,05)$ e menor renda per capita $(p=0,001)$. Conclusão: Podemos concluir que a IA esteve associada fortemente à aglomeração domiciliar e renda entre famílias de agricultores. Assim, as políticas públicas voltadas à saúde dos agricultores devem considerar a vulnerabilidade das famílias de baixa renda e com crianças e jovens na sua composição, possibilitando acesso à alimentação adequada.

Palavras-chave: segurança alimentar e nutricional; agricultor; avaliação nutricional.

\begin{abstract}
Background: In Brazil, it has been envisioned significant advances in food and nutrition policies. Despite this, the country has millions of people under conditions of Food Insecurity (FI), with recognized difficulties of access to adequate food. Objective: This research aimed to investigate the association between $\mathrm{FI}$ with anthropometric and socioeconomic statusof farmers of the municipalities in the Middle-South region of Ceará. Method: It is a cross-sectional study that used a socioeconomic questionnaire and the Brazilian Food Insecurity Scale to assess the situation of FI and the Body Mass Index (BMI) for assessing the nutritional status. The tests of Mann-Whitney and Chi-square were used, considering a significant $p$-value $(p<0.05)$. Results: 86 farmers participated in this research, including $39.5 \%$ of those who wereFI and $41 \%$ overweight. The association was identified between the situation of FI in families with the greatest number of individuals $(p<0.05)$, the presence of individuals aged under18 years $(p<0.05)$ and lower per capita income $(p=0.001)$. Conclusion: It could be conclude that the FI was strongly associated
\end{abstract}

Trabalho realizado pelo Centro Universitário de Juazeiro do Norte, Juazeiro do Norte (CE), Brasil.

Correspondência: Elida Mara Braga Rocha. E-mail: elidamara92@gmail.com

Fonte de financiamento: Próprio.

Conflito de interesses: nada a declarar.

Recebido em: Out. 14, 2018. Aceito em: Mar. 02, 2020
Este é um artigo publicado em acesso aberto (Open Access) sob a licença Creative Commons Attribution, que permite uso, distribuição e reprodução em qualquer meio, sem restrições desde que o trabalho original seja corretamente citado. 
to household crowding and income among farmers' families Thus, the public policies directed to the farmers' health should consider the vulnerability of low-income families and families with children as well as young people in their composition, enabling access to adequate food.

Keywords: food security; farmers; nutrition assessment.

\section{INTRODUÇÃO}

Alimentação e nutrição são indispensáveis para promoção e proteção da saúde, pois favorecem o desenvolvimento, crescimento e melhoria da qualidade de vida do ser humano'. E, nesse aspecto, o conceito de Segurança Alimentar e Nutricional (SAN) foi socialmente construído, abordando o Direito Humano à Alimentação Adequada (DHAA) como base norteadora, através da garantia de acesso ao alimento de modo regular e permanente, em qualidade e quantidade suficiente, sem comprometer outras necessidades, respeitando as práticas alimentares promotoras de saúde, a diversidade cultural, ambiental, econômica e socialmente sustentáveis².

Por sua vez, o conceito de Insegurança Alimentar (IA) está relacionado a um fenômeno complexo com dimensões econômicas, sociais, culturais e políticas de cada nação, atrelado com situações de vulnerabilidade social, exposição a determinados tipos de riscos e uma série de fatores que reduzem o nível de bem-estar das pessoas, comunidades e famílias ${ }^{3}$.

O primeiro pensador a denunciar e fazer grandes estudos sobre essa temática no país foi o médico Josué de Castro, em sua obra clássica Geografia da Fome, de 1946. Contudo, foi apenas a partir da segunda metade do século XX que o Brasil retomou o assunto, tendo como marco a I Conferência Nacional de Segurança Alimentar e a "Ação de Cidadania contra a Miséria, a Fome e pela Vida", liderada pelo sociólogo e ativista Betinho, nos anos de 1990, que semearam o caminho para inserção da SAN na agenda do governo brasileiro, e por meio da ll Conferência Nacional de Segurança Alimentar e Nutricional, em 2004, grandes propostas foram lançadas ${ }^{4}$, culminando dois anos depois na Lei Orgânica de Segurança Alimentar e Nutricional (LOSAN), que cria o Sistema Nacional de Segurança Alimentar e Nutricional (SISAN) 5 .

No mesmo ano, em 2006, foi instituída a Política Nacional de Agricultura Familiar, marco em que o Estado brasileiro reconhece a importância da produção agrícola local e sustentável tanto como mecanismo de desenvolvimento econômico quanto para garantia das condições plenas de $\mathrm{SAN}^{6}$. E nessa perspectiva, as famílias de agricultores ganharam espaço e reconhecimento institucional, ao serem consideradas como os principais agentes no processo de oferta de alimentos para a maioria dos brasileiros, portanto decisivos para garantia do DHAA no país.

A base do consumo alimentar dos brasileiros é constituída de produtos oriundos da agricultura familiar, o que torna inquestionáveis o papel e a importância desses atores na garantia e oferta de alimentos orgânicos, agroecológicos e nutritivos para as famílias brasileiras e para garantia da SAN?

Alinhado a isso, o Estado, por meio de políticas e programas públicos, fomenta ações e projetos direcionados a essas famílias produtoras, principalmente na perspectiva técnica e financeira, como o Garantia-Safra, o Seguro da Agricultura Familiar (SEAF), o Programa Nacional de Crédito Fundiário (PNCF), a Política Nacional de Assistência Técnica e Extensão Rural (Pnater), o Programa de Aperfeiçoamento da Agricultura Familiar (Pronaf) e o Programa de Aquisição de Alimentos (PAA) ${ }^{8}$.

Entretanto, no que se refere à oferta de bens e serviços de saúde para as populações do campo, das águas e das florestas, muitos desafios ainda não foram superados ou estão muito aquém dos anseios desses atores, por razões desde a falta de intersetorialidade das políticas públicas até diversas limitações orçamentárias nos planos governamentais de saúde. Somase a essa problemática o fato da IA se configurar como um significativo problema nacional, principalmente, no Norte e Nordeste do país, visto que essas regiões apresentam os maiores percentuais de pessoas em $1 \mathrm{~A}^{9}$, sendo um território propício para a investigação das condições de alimentação e nutrição dos agricultores familiares.

Sendo assim, este trabalho busca responder os seguintes questionamentos: Será que essas famílias do campo estão em condições de segurança alimentar e nutricional? Existe uma 
relação entre perfil socioeconômico, antropométrico e Insegurança Alimentar e Nutricional entre agricultores familiares?

Nessa perspectiva, o objetivo deste trabalho é associar a percepção de Insegurança Alimentar de agricultores do Cariri cearense com o perfil socioeconômico e antropométrico, abordando as questões de saúde e nutrição dos mesmos, por não os considerar apenas como agentes produtores de alimentos, mas também como atores indispensáveis no desenvolvimento local e no empoderamento das famílias do campo.

\section{MÉTODO}

Trata-se de uma pesquisa transversal, realizada nos meses de agosto e setembro de 2016, nas feiras, mercados populares de alimentos e associações de agricultores/produtores de municípios da Região Metropolitana do Cariri, Ceará. Algumas dessas feiras integram a Rede de Feiras Agroecológicas do Cariri. O lócus da pesquisa contemplou as feiras realizadas nos municípios de Crato, Juazeiro do Norte e Barbalha, conhecido por triângulo Crajubar.

O cálculo amostral considerou a última prevalência de insegurança alimentar moderada e grave do estado do Ceará ${ }^{9}$, um a de $5 \%$, poder de $80 \%$ ( $\left.\beta-1\right)$ e um acréscimo de $10 \%$ para possíveis perdas.

Os sujeitos do estudo foram os agricultores participantes de feiras, mercados populares de alimentos e associações de agricultores/produtores de municípios da Região Metropolitana do Cariri, que se enquadraram nos seguintes critérios de inclusão: maiores de 18 anos de idade; estarem vinculados ao cadastro de feirantes da região do Cariri e residência nos municípios de Barbalha, Juazeiro do Norte ou Crato. Foram excluídos da análise aqueles que não completaram a avaliação antropométrica ou entrevista.

Foi utilizado para coleta de dados socioeconômicos e demográficos um questionário pré-codificado, abordando as variáveis: idade; gênero; escolaridade em anos completos de estudo do chefe da família; composição familiar (número de integrantes maiores e menores de 18 anos de idade); informações sobre participação em programas sociais e renda familiar mensal - por serem conhecidos como importantes condicionantes da Insegurança Alimentar. Além da coleta de dados sobre acesso à luz elétrica e às condições de saneamento básico, referentes ao abastecimento da água, tratamento doméstico da água para beber, tratamento do esgoto e coleta de lixo.

A Escala Brasileira de Insegurança Alimentar (EBIA) foi utilizada para investigação da insegurança alimentar e nutricional da família do agricultor. Esse instrumento é reconhecido e validado em amostra intencional de populações urbanas de quatro cidades brasileiras e em populações rurais de quatro regiões nacionais. Cada resposta afirmativa do questionário representa 1 ponto, onde a pontuação da escala, sua soma, varia em uma amplitude de 0 a 15 pontos, que classifica as famílias em uma das quatro categorias, segundo os escores de pontuação. Para domicílios com pelo menos um morador menor de 18 anos de idade: Segurança Alimentar e Nutricional (SAN) com 0 pontos; IA leve com 1 a 5 pontos; IA moderada com 6 a 10 pontos e IA grave com 11 a 15 pontos. Já para domicílios com apenas moradores de 18 anos ou mais de idade: 0 pontos para SAN; 1 a 3 pontos para IA leve; 4 a 6 pontos para IA moderada e 7 a 9 pontos para IA grave. A classificação de famílias em IA leve é referente à preocupação de ficar sem alimento para garantir o futuro imediato, ou comprometimento qualitativo da dieta por limitação financeira; IA moderada com restrição qualitativa e quantitativa na dieta entre adultos; e IA grave, onde a restrição na dieta é qualitativa e quantitativa entre adultos e crianças ${ }^{10}$.

Para avaliação antropométrica utilizaram-se as medidas de peso e altura, segundo as normas técnicas da Organização Mundial da Saúde, seguida pelo Ministério da Saúde ${ }^{11}$, sendo solicitado ao participante o seu posicionamento descalço, com o mínimo de roupa possível para aferição de peso e altura. Os instrumentos utilizados para mensuração dessas medidas foram uma balança digital da marca Britânica -BE3 ${ }^{\circledR}$, com capacidade de $150 \mathrm{~kg}$ e intervalos de $100 \mathrm{~g}$ e um antropômetro da marca Avanutri ${ }^{\circledR}$ apoiado na parede, de leitura lateral, com extensão de 0 a 2 m e graduação em milímetros. 
A análise da situação nutricional foi realizada utilizando o Índice de Massa Corporal (IMC), classificado de acordo com os critérios para adultos de baixo peso aqueles com IMC $<18,5 \mathrm{~kg} / \mathrm{m}^{2}$; eutrofia com IMC entre 18,5 e 24,9 kg/m²; excesso de peso com IMC entre 25 e $29,9 \mathrm{~kg} / \mathrm{m}^{2}$; e indivíduos obesos com IMC $\geq 30 \mathrm{~kg} / \mathrm{m}^{2}$, segundo a Organização Mundial da Saúde ${ }^{12}$. Já para idosos ${ }^{13}$ : baixo peso com IMC $<22 \mathrm{~kg} / \mathrm{m}^{2}$; eutrofia com IMC entre 22 e $27 \mathrm{~kg} / \mathrm{m}^{2}$ e sobrepeso para IMC $>27 \mathrm{~kg} / \mathrm{m}^{2}$.

As análises estatísticas foram realizadas com auxílio do software Statistical Package for the Social Sciences (SPSS), versão 20.0 for Windows. Os dados foram descritos na forma de medida de proporção (frequência e prevalência), medidas de tendência (médias e medianas) e medidas de dispersão. A análise dos fatores que influenciam a prevalência de insegurança alimentar e nutricional em famílias de agricultores foi realizada em duas etapas. Na primeira etapa, foi analisado cada um dos determinantes da insegurança alimentar e nutricional, procedendo à análise descritiva das informações que caracterizam a população estudada e à análise exploratória para a correção das inconsistências. Variáveis contínuas foram testadas para normalidade através de histogramas e do teste de Kolmogorov-Smirnov. Na segunda etapa, o teste qui-quadrado foi realizado para associação das variáveis categóricas independentes com a situação de insegurança alimentar (leve, moderada e grave), já para comparação de medianas entre as variáveis contínuas foi utilizado o teste de Mann-Whitney, considerando como estatisticamente significante $p<0,05$.

A pesquisa foi aprovada pelo Comitê de Ética em Pesquisa da Faculdade de Juazeiro do Norte, sob o número de parecer 1.692.064, atendendo as normas regulamentares de pesquisa envolvendo seres humanos, conforme recomenda a Resolução $n^{0} 466 / 12$, do Conselho Nacional de Saúde ${ }^{14}$.

\section{RESULTADOSE DISCUSSÃO}

\section{Caracterização da amostra}

Foram entrevistados 86 agricultores, em sua maioria do gênero masculino $(70,9 \%)$, entre 30 e 59 anos de idade (58,1\%), com escolaridade de 1 a 5 anos de estudos (47,7\%), apresentando renda per capita mensal < $1 / 2$ salário mínimo (56,9\%), além de $44 \%$ dos entrevistados mencionarem receber um ou mais tipos de benefícios federais (Tabela 1), sendo que o valor do benefício social apresentou uma mediana mensal de $\mathrm{R} \$ 178$, com variação entre os tipos de programa.

Dentre os seis programas federais investigados, o mais frequente foi o Programa Bolsa Família, com $42,4 \%$ dos agricultores sendo beneficiados com valor médio de $\mathrm{R} \$ 112$, seguido do Programa Cisterna (42\%), Pronaf (25\%), Garantia Safra $(17,6 \%)$ com valor médio de R\$71, PAA (14,3\%), com valor médio de R\$ 410, e Programa Nacional de Alimentação Escolar - PNAE $(10,1 \%)$ com valor médio de $\mathrm{R} \$ 452$.

Quanto às condições de saneamento básico, aproximadamente $70 \%$ dos entrevistados contam com coleta municipal de lixo e a existência de fossa séptica no domicílio. A água utilizada para beber em $62,8 \%$ das famílias advém de poço, cacimba ou barreiro, sendo que $70,9 \%$ dos agricultores destacaram que existe algum tipo de tratamento de água (Tabela 1).

\section{Situação nutricional}

Na Figura 1 estão ilustrados os resultados da EBIA que revelou 23,3\% das famílias em condições de insegurança alimentar (leve, moderada e grave) e o restante em condições de segurança alimentar e nutricional.

O estudo obteve resultados semelhantes com os da Pesquisa Nacional por Amostra de Domicílios (PNAD), de 2013, que identificou IA em 22,6\% dos domicílios do país, sendo 3,2\% em IA grave9. Por outro lado, discorda dos resultados encontrados na área rural da zona da mata nordestina ${ }^{15}$, em que $88 \%$ das famílias entrevistadas estavam em condições de IA. 
Tabela 1. Caracterização socioeconômica das famílias de agricultores da Região Metropolitana do Cariri Cearense, Ceará-CE, Brasil, 2016

\begin{tabular}{|c|c|c|}
\hline Variáveis & $\mathbf{n}$ & $\%$ \\
\hline \multicolumn{3}{|l|}{ Gênero } \\
\hline Masculino & 61 & 70,9 \\
\hline Feminino & 25 & 29,1 \\
\hline \multicolumn{3}{|l|}{ Idade } \\
\hline$<30$ anos & 12 & 14,0 \\
\hline 30 a 59 anos & 50 & 58,1 \\
\hline$\geq 60$ anos & 24 & 27,9 \\
\hline \multicolumn{3}{|l|}{ Escolaridade } \\
\hline Sem estudo & 7 & 8,1 \\
\hline 1 a 5 anos de estudo & 41 & 47,7 \\
\hline 6 a 9 anos de estudo & 13 & 15,1 \\
\hline$>9$ anos de estudo & 25 & 29,1 \\
\hline \multicolumn{3}{|l|}{ Renda per capita } \\
\hline$<1 / 2$ S.M.* & 49 & 56,9 \\
\hline 1/2 a 1 S.M. & 23 & 26,7 \\
\hline$\geq 1$ S.M. & 9 & 10,5 \\
\hline \multicolumn{3}{|l|}{ Recebe benefício social } \\
\hline Sim & 37 & 44,0 \\
\hline Não & 47 & 66,0 \\
\hline \multicolumn{3}{|l|}{ Água } \\
\hline Rede pública & 28 & 32,6 \\
\hline Poço/cacimba/barreiro & 54 & 62,8 \\
\hline Outro & 4 & 4,7 \\
\hline \multicolumn{3}{|l|}{ Tratamento de água } \\
\hline Sim & 61 & 70,9 \\
\hline Não & 25 & 29,1 \\
\hline \multicolumn{3}{|l|}{ Lixo } \\
\hline Coleta municipal & 58 & 68,2 \\
\hline Incinerado & 23 & 27,1 \\
\hline A céu aberto & 4 & 4,7 \\
\hline \multicolumn{3}{|l|}{ Esgoto } \\
\hline Fossa séptica & 59 & 68,6 \\
\hline Fossa rudimentar & 20 & 23,3 \\
\hline Vala/a céu aberto & 7 & 8,2 \\
\hline
\end{tabular}

*S.M. = Salário Mínimo de R\$ 880,00, 2016.

Condições de Segurança Alimentar e Nutricional de Agricultores

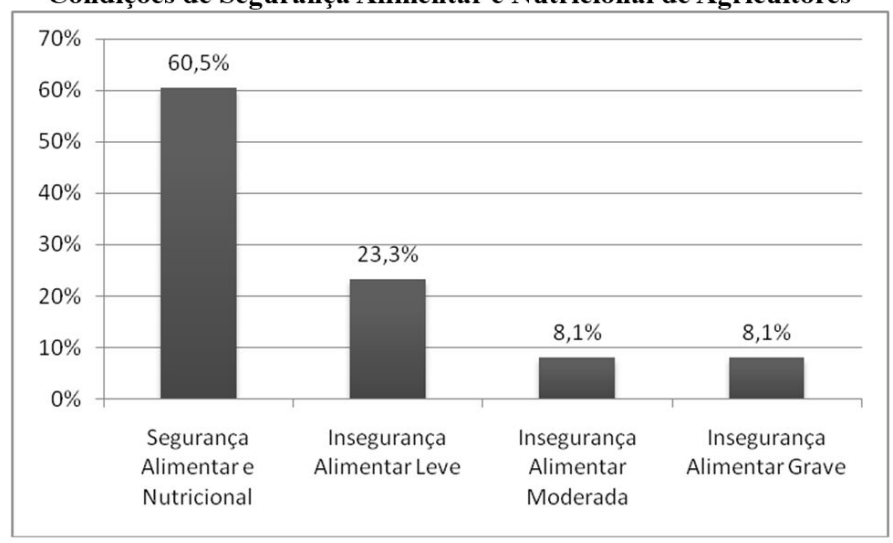

Figura 1. Distribuição percentual de agricultores segundo a Escala Brasileira de Insegurança Alimentar e Nutricional-EBIA, Região Metropolitana do Cariri Cearense, Ceará-CE, Brasil, 2016 
A avaliação antropométrica demonstrou que em $47,7 \%$ dos agricultores apresentavam excesso de peso ( $32,6 \%$ sobrepeso e $15,1 \%$ obesidade) e $5,8 \%$ desnutrição, sendo dentre estes todos agricultores idosos.

As pessoas em condição de IA geralmente consomem uma alimentação reduzida em variedade e nutrientes e monótona ${ }^{16}$, o que contribui para o surgimento de obesidade e doenças crônicas não transmissíveis, sendo que as mais comuns são diabetes mellitus, hipertensão arterial sistêmica e doenças cardiovasculares ${ }^{17}$.

A elevada prevalência de excesso de peso também foi destacada na Pesquisa de Orçamento Familiar - POF 2008-2009-, onde observa-se o aumento paulatino desde a década de 70 e hoje atinge praticamente metade dos brasileiros ${ }^{18}$.

Tal contexto nutricional se deve principalmente ao consumo de dietas ricas em açúcares e gorduras, alimentos ultraprocessados, refrigerantes e outros alimentos hipercalóricos intensifica esse processo. Por outro lado, esse comportamento veio interligado com a diminuição no consumo de fibras, carboidratos complexos, alimentos naturais e legumes ${ }^{19}$.

A associação das variáveis socioeconômicas com a situação de SAN e IA permitiu verificar a existência de uma diferença estatisticamente significante entre os agricultores quanto à renda per capita $(p=0,001)$ e número de membros da família $(p=0,005)$, indicando que o agricultor com menor renda e/ou maior número de integrantes no domicílio possui maior risco de estar em IA (Tabela 2).

Tabela 2. Associação das variáveis contínuas socioeconômicas e nutricionais com a situação de Segurança e Insegurança Alimentar de agricultores da Região Metropolitana do Cariri Cearense, Ceará-CE, Brasil, 2016

\begin{tabular}{lcccc} 
& \multicolumn{1}{c}{ SAN } & IA & p \\
\cline { 2 - 3 } Idade & mediana (min-max) & & mediana (min-max) & \\
\hline Renda per capita & $51,5(22,0-81,0)$ & & $42,5(20,0-81,0)$ & 0,06 \\
\hline Valor benefícios & $440,0(70,0-1760)$ & $220,0(0-660,0)$ & $\mathbf{0 , 0 0 1}$ \\
\hline No de moradores & $190,0(75,0-4000,0)$ & $152,0(28,0-880,0)$ & 0,08 \\
\hline No $<18$ anos de idade & $3(1-8)$ & $5(2-8)$ & $\mathbf{0 , 0 0 5}$ \\
\hline IMC & $1(1-3)$ & $2(1-3)$ & 0,14 \\
\hline
\end{tabular}

Teste de Mann-Whitney. SAN: Segurança Alimentar; IA: Insegurança Alimentar; IMC: Índice de Massa Corporal

Vasconcelos et al. ${ }^{17}$ encontraram uma associação positiva entre classe econômica $D$ e renda per capita menor do que $1 / 4$ do salário mínimo com IA. Essa relação indica que o grau de carência alimentar está diretamente associado ao nível de renda familiar, na medida em que existe comprometimento da qualidade de vida, acesso a bens e serviços essenciais devido às limitações monetárias, além do exercício da cidadania e trabalho³. Dessa forma, pertencer à classe socioeconômica mais desfavorável é um indicativo de risco para IA. O que vem a confirmar esse entendimento no que diz respeito à renda, pois uma vez que é um fator determinante na aquisição de gêneros alimentícios está, portanto, diretamente interligada à adequação no consumo alimentar familiar".

Mesmo que não tenha sido possível verificar associação de IA com recebimento de benefícios sociais, sabe-se que a maioria desses programas enquadram-se nas propostas das políticas públicas redistributivas, em especial a transferência de renda como mecanismo de melhoria das condições sociais, repercutindo em um ganho de qualidade na busca pela segurança alimentar efetiva das famílias com algum tipo de vulnerabilidade ${ }^{20}$. Também para esses autores e Burlandy ${ }^{21}$, os programas de transferências de renda não são suficientes para atender a complexidade que envolve esse fenômeno no sentido das melhorias das condições socioeconômicas. 
Outro aspecto a ser destacado é o fato de a aglomeração domiciliar influenciar na situação de IA, permitindo discutir essa variável como um fator determinante na vulnerabilidade alimentar, na medida em que está diretamente relacionado com a despesa da família com alimentação e demais obrigações, ou seja, quanto maior o número de pessoas, maior será o impacto no orçamento familiar ${ }^{22,23}$.

A relevância da composição familiar na determinação de IA entre famílias de agricultores deve-se ao fato de que a presença de menores de 18 anos de idade no domicílio está fortemente relacionada à $\mathrm{IA}, \mathrm{p}<0,001$ (Tabela 3 ).

Tabela 3. Associação das variáveis categóricas socioeconômicas e sanitárias com a situação de Segurança e Insegurança Alimentar de agricultores da Região Metropolitana do Cariri Cearense, Ceará-CE, Brasil, 2016

\begin{tabular}{|c|c|c|c|c|}
\hline \multirow{2}{*}{ Variáveis } & Total & IA $(n=34)$ & SAN $(n=52)$ & \multirow{2}{*}{$\mathbf{p}$} \\
\hline & $\mathbf{n}$ & $\%$ & $\%$ & \\
\hline \multicolumn{5}{|l|}{ Gênero } \\
\hline Feminino & 25 & 56,0 & 44,0 & 0,05 \\
\hline Masculino & 61 & 32,8 & 67,2 & \\
\hline \multicolumn{5}{|l|}{ Escolaridade } \\
\hline Sem estudo & 7 & 57,1 & 42,9 & 0,17 \\
\hline 1 a 5 anos de estudo & 41 & 36,6 & 63,4 & \\
\hline 6 a 9 anos de estudo & 13 & 61,5 & 38,5 & \\
\hline$>9$ anos de estudo & 25 & 28,0 & 72,0 & \\
\hline \multicolumn{5}{|c|}{ Menores de $<18$ anos no domicílio } \\
\hline Pelo menos um & 48 & 70,8 & 29,2 & $<0,001$ \\
\hline Nenhum & 38 & 0,0 & 100,0 & \\
\hline \multicolumn{5}{|l|}{ Água } \\
\hline Rede pública & 28 & 53,6 & 46,4 & 0,06 \\
\hline Outros & 58 & 32,8 & 67,2 & \\
\hline \multicolumn{5}{|l|}{ Lixo } \\
\hline Coleta municipal & 58 & 39,7 & 60,3 & 0,82 \\
\hline Outros & 27 & 37,0 & 63,0 & \\
\hline \multicolumn{5}{|l|}{ Esgoto } \\
\hline Fossa séptica & 59 & 40,7 & 59,3 & 0,75 \\
\hline Outros & 27 & 37,0 & 63,0 & \\
\hline
\end{tabular}

Teste qui-quadrado; SAN: Segurança Alimentar; IA: Insegurança Alimentar

Em seus estudos, Anschau et al. ${ }^{24}$ destacaram que a proporção de segurança alimentar $(54,2 \%)$ é em média duas vezes maior em famílias sem crianças e adolescentes do que naquelas com menores de idade no domicílio. Em conformidade ao retratado em um inquérito nacional, em que os indivíduos que apresentaram pelo menos um menor de 18 anos de idade em sua composição familiar possuíam maior prevalência de insegurança alimentar ${ }^{9}$. E nesse sentido, os achados no presente trabalho também foram encontrados nos estudos de Panigasi et al. ${ }^{22}$ e Souza et al. ${ }^{23}$, onde a IA grave e moderada é mais comum em famílias numerosas e com precárias condições socioeconômicas e de saneamento básico. 
Uma associação que merece destaque refere-se à relação de IA e gênero (Tabela 3), uma vez que os dados demonstraram que a IA está mais presente na vida das mulheres $(56 \%$, $p=0,05)$. Esse resultado possivelmente vincula-se às funções sociais exercidas pela mulher, principalmente no núcleo familiar. Há uma série de fatores que podem contribuir para tal fato dos quais podemos mencionar: as responsabilidades domésticas; a inserção no mercado de trabalho e ao mesmo tempo a manutenção do lar e o cuidado com os filhos ${ }^{25}$. E nesse contexto, Rocha e Rigotto ${ }^{26}$ apontam que a mulher na cadeia produtiva encontrara-se em grande vulnerabilidade, situação essa que impacta negativamente sobre a sua saúde, seja como consequência da divisão sexual do trabalho ou pela baixa renda, o fato é que mulheres trabalhadoras do campo sofrem risco psico, social e mental devido às desigualdades de gênero.

Por outro lado, distanciando-se do resultado esperado, a pesquisa não apontou relação entre a IA e o perfil antropométrico dos entrevistados. O que pode ser explicado pelo fato de que a antropometria, por ser um método indireto para medir a IA, não consegue associar por si só todas as relações de consumo alimentar com o estado nutricional, uma vez que a IA está interligada ao grau de vulnerabilidade socioeconômica das famílias (renda per capita) e pode não ter relação direta com a realidade do estado nutricional da população em transição nutricional ${ }^{27}$, sendo assim, mesmo considerado um hábil indicador nutricional, a antropometria parece não manter diálogo direto com a IA, visto que a mensuração das dimensões ponderais não reflete corretamente a qualidade ou quantidade de alimentos consumidos e muito menos o acesso regular à alimentação adequada e digna $a^{3,28}$.

A exemplo disso, Peña e Bacallao ${ }^{29}$ explicam que a obesidade não é sinônimo de alta renda e poder de compra e, portanto, de acesso à alimentação. Pelo contrário, há um aumento de sobrepeso na população de baixa renda pela compra de alimentos mais baratos e de baixa qualidade nutritiva.

Assim, acreditamos que a existência da relação entre IA e perfil antropométrico na pesquisa transversal, tipicamente quantitativa, pode não ter sido expressiva a ponto de atingir o grau de significância $(p<0,05)$ por tratar-se de um indicador indireto da IA, haja vista que a mensuração da situação nutricional sofre influência de diversos fatores ${ }^{27}$.

Diante desse contexto, Magalhães ${ }^{30}$ aponta a necessidade de promoção de novos acordos e modelos de desenvolvimento sustentável, com outra abordagem paradigmática, sendo que Lüchmann ${ }^{31}$ menciona que um caminho para inserção das demandas públicas no âmbito da segurança alimentar e nutricional passará necessariamente pelo fortalecimento dos conselhos gestores e de direitos enquanto Instituições Participativas (IPs), onde o controle por meio da participação social pode ser potencializado exponencialmente, além de fomentar a construção e existência de políticas públicas efetivas.

A garantia do DHAA e da Soberania Alimentar requer ações e políticas públicas transversais, sistematizadas com base em indicadores concretos, com vistas a contemplar as dimensões múltiplas desse fenômeno, assim a transversalidade e intersetorialidade são termos fundamentais nesse processo $0^{5,32}$.

Nesse contexto, a agricultura familiar é indispensável nos processos e políticas públicas de SAN. Marques et al. ${ }^{33}$, em um estudo de caso realizado no município de Araripe-CE com agricultores, destacaram o estado como ator central nests processo a partir da compra direta/ mercado institucional, como no Programa Nacional de Alimentação Escolar (PNAE). Mas, por outro lado, esses autores também enfatizaram as dificuldades dos pequenos e médios agricultores em acessar esse mercado, desde a capacitação técnica até mesmo a organização política, em associações e cooperativas. E em consonância com esse discurso, Delgado e Bergamas $\mathrm{CO}^{8}$ destacam que os agricultores familiares são agentes fundamentais na garantia da SAN, mas para isso são necessários mecanismos e ações governamentais de fomento e desenvolvimento desses atores, tanto na capacitação como no acompanhamento contínuo pelos órgãos responsáveis. E a identificação dos determinantes das situações de insegurança alimentar viabiliza a construção de políticas públicas efetivas para esse problema, sendo que esses fatores estão relacionados principalmente com a pobreza e desigualdade social ${ }^{34}$.

O presente estudo apresentou algumas limitações. Como já foi mencionado em outros pontos, este trabalho não contempla todas as dimensões que envolvem as condições 
de insegurança alimentar e nutricional. Uma limitação que deve ser citada refere-se à confiabilidade nas respostas dos agricultores o local de coleta e a percepção dos mesmos sobre o trabalho podem ter influenciado diretamente nos resultados deste estudo. Além disso, os estudos transversais permitem apenas um recorte temporal pequeno das condições, dos atores sociais analisados, o que pode impedir a abstração de outros fenômenos do universo em questão, já que as variáveis causa e efeito são mensuradas ao mesmo tempo. Apesar disso, os resultados definem as características da amostra em estudo, podem revelar associações de interesse para outros estudos, e o desenho da pesquisa apresenta inúmeras vantagens, como a otimização de recursos humanos e materiais, além de possibilitar a formulação de hipóteses para analisar a rede de causalidade.

Outra limitação foi a impossibilidade de realizar a coleta de consumo alimentar habitual; tal indicador permitiria investigar melhor quais os alimentos mais consumidos por famílias de agricultores em segurança e insegurança alimentar. Mesmo que a EBIA seja estruturada na percepção do indivíduo sobre a escassez e falta de alimentos, envolvendo aspectos de inadequação nutricional tanto qualitativa e quantitativamente ${ }^{3,10,28}$, deve-se considerar a complexidade envolvida nessa área, sendo importante a realização de outros estudos com abordagem longitudinal e que contemplem outros fatores, tais como frequência do consumo alimentar, recordatório alimentar, gasto da família com alimentação.

Estudos futuros poderiam avaliar o consumo alimentar de agricultores, haja vista que a sociedade contemporânea, com o advento das mudanças nos padrões alimentares, de certa forma perdeu a preocupação com a fome extrema e escassez dos alimentos, mas aumentou significativamente o acesso e consumo de alimentos industrializados, hipercalóricos, ultraprocessados, ricos em gordura e sódio. Seria importante investigar se as famílias de agricultores também seguiam a mesma tendência alimentar, e se tal comportamento aumentaria o risco de excesso de peso, diretamente relacionado com as principais doenças crônicas não transmissíveis. Osório et al. ${ }^{35}$ destacam as diferenças existentes na ingestão de carnes, frutas e leite nas famílias com IA numa pesquisa feita na Zona da Mata e no Semiárido do Nordeste, onde foi encontrado baixo consumo de tubérculos e raízes, principalmente nas famílias com maior vulnerabilidade social.

Dessa forma, o fenômeno da transição nutricional em curso e as mudanças nos padrões alimentares dos brasileiros parecem estar em convergência também para índices elevados de insegurança alimentar, portanto é importante adotar outros instrumentos e mecanismos de avaliação das famílias e dos indivíduos, no sentido de abordar desde as características mais psicológicas, sociais, até os hábitos alimentares e a composição nutricional da dieta.

\section{CONSIDERAÇÕES FINAIS}

Os resultados permitiram aprofundar o debate sobre algumas dimensões da insegurança alimentar e nutricional, além de possíveis associações. Evidenciou-se uma relação estatisticamente comprovada entre IA e renda per capita, número de moradores no domićlio, principalmente em famílias com menores de 18 anos de idade. Por outro lado, mesmo sem encontrar uma associação estatisticamente significante, algumas variáveis parecem ter uma relação importante com IA em agricultores, como é caso do estado nutricional, idade e gênero, o que pode abrir espaço para outras investigações nesse sentido.

Assim, as políticas públicas devem promover estratégias de educação nutricional efetivas entre as famílias de agricultores, principalmente nas de baixa renda e que possuem crianças e jovens na sua composição, possibilitando condições de escolhas saudáveis além do acesso ao alimento. Iniciativas que busquem ampliar a rede de cuidados ou mesmo sinalizar para novas necessidades desses atores, os agricultores, no âmbito da saúde e da SAN, são importantes na medida em que fortalecem essas unidades produtoras de alimentos e garantem a integralidade em saúde e o DHAA. 


\section{REFERÊNCIAS}

1. Brasil. Ministério da Saúde. Secretaria de Atenção à Saúde. Política nacional de alimentação e nutrição. Brasília: MS; 2011.

2. Brasil. Ministério do Desenvolvimento Social e Combate à Fome. Conselho Nacional de Segurança Alimentar e Nutricional - Consea. II Conferência Nacional de Segurança Alimentar e Nutricional. Relatório Final. [Internet]. Brasília: MDS; 2004 [citado em 2016 abr 10]. Disponível em: http://www.mds.gov.br/ saladeimprensa/eventos/seguranca-alimentar-e-nutricional/ii-conferencia-nacional-de-segurancaalimentar-e-nutricional-1/sobre-o-evento/relatorio\%20final.PDF/download

3. Kepple AW, Segall-Corrêa M. Conceituando e medindo segurança alimentar e nutricional. Ciênc. Saúde Coletiva. 2011;16(1):187-99. http://dx.doi.org/10.1590/S1413-81232011000100022.

4. Maluf RSJ. Segurança alimentar e nutricional. Petrópolis: Vozes; 2007.

5. Brasil. Lei n 11.346, de 15 de setembro de 2006. Dispõe sobre a criação do Sistema Nacional de Segurança Alimentar e Nutricional - SISAN com vistas em assegurar o direito humano à alimentação adequada e dá outras providências. Diário Oficial da União [Internet], Brasília, 15 de setembro de 2006 [citado em 2019 nov 11]. Disponível em: http://www.planalto.gov.br/ccivil_03/_Ato2004-2006/2006/Lei/L11346.htm

6. Brasil. Lei no 11.326, de 24 de julho de 2006. Estabelece as diretrizes para a formulação da Política Nacional da Agricultura Familiar e Empreendimentos Familiares Rurais. Diário Oficial da União [Internet], Brasília, 24 de julho de 2006 [acesso em 2016 ago 20]. Disponível em: http://www.planalto.gov.br/ccivil_03/_Ato20042006/2006/Lei/L11326.htm

7. Hoffmann H. A agricultura familiar produz $70 \%$ dos alimentos consumidos no Brasil? Segur Aliment Nutr. 2014;21(1):417-21. http://dx.doi.org/10.20396/san.v21i1.1386.

8. Delgado GC, Bergamasco SMPP. organizador. Agricultura familiar brasileira: desafios e perspectivas de futuro [Internet]. Brasília: Ministério do Desenvolvimento Agrário; 2017. [citado em 2018 ago 19]. Disponível em: http://www.mda.gov.br/sitemda/sites/sitemda/files/user_img_1756/Agricultura\%20 Familiar_WEB_LEVE.pdf

9. Brasil. Instituto Brasileiro de Geografia e Estatística. Pesquisa Nacional por Amostra de Domicílios (PNAD): Segurança alimentar [Internet]. Rio de Janeiro: IBGE; 2013 [citado em 2016 set 25]. Disponível em: https:// biblioteca.ibge.gov.br/visualizacao/livros/liv91984.pdf

10. Segall-Corrêa AM, Escamilla RP, Sampaio MFA, Marin-Leon L, Panigassi G, Maranha LK et al. Acompanhamento e avaliação da segurança alimentar de famílias brasileiras: validação de metodologia e de instrumento de coleta de informação Urbano/Rural. Relatório técnico [Internet]. Brasília: Ministério da Saúde, Organização Pan-Americana da Saúde; 2004 [citado em 2016 dez 16]. Disponível em: http://bvsms.saude.gov.br/bvs/ publicacoes/vru_unic.pdf.

11. Brasil. Ministério da Saúde (MS). Secretaria de Atenção à Saúde. Departamento de Atenção Básica. Orientações para a coleta e análise de dados antropométricos em serviços de saúde: Norma Técnica do Sistema de Vigilância Alimentar e Nutricional - SISVAN [Internet]. Brasília: MS; 2011 [citado em 2016 ago 19]. Disponível em: http://189.28.128.100/dab/docs/portaldab/publicacoes/orientacoes_coleta_analise_ dados_antropometricos.pdf

12. World Health Organization. Physical status: the use and interpretation of anthropometry. Geneva: WHO; 1995.

13. Lipschitz DA. Screening for nutritional status in the elderly. Prim Care. 1994;21(1):55-67. PMid:8197257.

14. Brasil. Conselho Nacional de Saúde. Resolução ${ }^{\circ} 466$, de 12 de dezembro de 2012. Normas para pesquisa envolvendo seres humanos. Diário Oficial da União, Brasília, 12 de dezembro de 2012.

15. Oliveira JS, Lira PIC, Maia SR, Sequeira LAS, Amorim RCA, Batista Filho M. Insegurança alimentar e estado nutricional de crianças de Gameleira, zona da mata do Nordeste brasileiro. Rev Bras Saúde Mater Infant. 2010;10(2):237-45. http://dx.doi.org/10.1590/S1519-38292010000200011.

16. Panigassi G, Segall-Corrêa AM, Marin-León L, Pérez-Escamilla R, Maranha LK, Sampaio MFA. Insegurança alimentar intrafamiliar e perfil de consumo de alimentos. Rev Nutr. 2008;21(Suppl.):135-44. http://dx.doi. org/10.1590/S1415-52732008000700012.

17. Vasconcelos SML, Torres NCP, Silva PMC, Santos TMP, Silva JVL, Omena CMB, et al. Insegurança alimentar em domicílios de indivíduos portadores de hipertensão e/ou diabetes. Int. j. cardiovasc. Sci. 2015;28(2):11421.

18. Brasil. Ministério do Planejamento, Orçamento e Gestão. Instituto Brasileiro de Geografia e Estatística. Pesquisa de Orçamentos Familiares 2008-2009. Antropometria e Estado Nutricional de Crianças, Adolescentes e Adultos no Brasil [Internet]. Rio de Janeiro: IBGE; 2010 [citado em 2017 abr 03]. Disponível em: https://biblioteca.ibge.gov.br/visualizacao/livros/liv45419.pdf 
19. Canella DS, Louzada MLC, Claro RM, Costa JC, Bandoni DH, Levy RB, et al. Consumo de hortaliças e sua relação com os alimentos ultraprocessados no Brasil. Rev Saude Publica. 2018;52:50. http://dx.doi. org/10.11606/S1518-8787.2018052000111. PMid:29791530.

20. Segall-Corrêa AM, Marin-Leon L, Helito H, Pérez-Escamilla R, Santos LMP, Paes-Sousa R. Transferência de renda e segurança alimentar no Brasil: análise dos dados nacionais. Rev Nutr. 2008;21(Suppl.):39-51. http://dx.doi.org/10.1590/S1415-52732008000700005.

21. Burlandy L. Transferência condicionada de renda e segurança alimentar e nutricional. Ciênc. Saúde Coletiva. 2007;12(6):1441-51. http://dx.doi.org/10.1590/S1413-81232007000600007.

22. Panigassi G, Segall-Corrêa AM, Marín-León L, Pérez-Escamilla R, Sampaio MF, Maranha LK. Insegurança alimentar como indicador de iniqüidade: análise de inquérito populacional. Cad Saude Publica. 2008;24(10):2376-84. http://dx.doi.org/10.1590/S0102-311X2008001000018. PMid:18949239.

23. Souza NN, Dias MM, Sperandio N, Franceschini SCC, Priore SE. Perfil socioeconômico e insegurança alimentar e nutricional de famílias beneficiárias do Programa Bolsa Família no município de Viçosa, Estado de Minas Gerais, Brasil, em 2011: um estudo epidemiológico transversal. Epidemiol Serv Saude. 2012;21(4):655-62. http://dx.doi.org/10.5123/S1679-49742012000400015.

24. Anschau FR, Matsuo T, Segall-Corrêa AM. Insegurança alimentar entre beneficiários de programas de transferência de renda. Rev Nutr. 2012;25(2):177-89. http://dx.doi.org/10.1590/S1415-52732012000200001.

25. Lima ACO, Lima RSV, Silva JMA. Gênero Feminino, contexto histórico e segurança alimentar. Demetra. 2016;11(3):789-802.

26. Rocha MM, Rigotto RM. Produção de vulnerabilidades em saúde: o trabalho das mulheres em empresas agrícolas da Chapada do Apodi, Ceará. Saúde Debate. 2017;41(N. spe 2), 63-79.

27. Rocha EMB, Lima RT, Diniz DB, Almeida PC. Situação Nutricional de crianças em munícipio de privilegiado Índice de Desenvolvimento Humano do semiárido brasileiro e sua relação com a Insegurança Alimentar. Segur Aliment Nutr. 2012;19(2):17-29. http://dx.doi.org/10.20396/san.v19i2.8634608.

28. Perez-Escamilla R, Segall-Côrrea AM. Food insecurity measurement and indicators. Rev Nutr. 2008;21(Suppl.):15s-26s. http://dx.doi.org/10.1590/S1415-52732008000700003.

29. Peña M, Bacallao J. La obesidad em la pobreza: un nuevo reto para la salud pública. Washington: OPAS; 2000.

30. Magalhães R. Avaliação de políticas e iniciativas públicas de segurança alimentar e nutricional: dilemas e perspectivas metodológicas. Ciênc. Saúde Coletiva. 2014;19(5):1339-46. http://dx.doi.org/10.1590/141381232014195.12202013.

31. Lüchmann LHH. Os conselhos gestores de políticas públicas: desafios do desenho institucional. Ciências Sociais Unisinos. 2002;38n(161):43-79.

32. Siqueira RL, Fonseca DA, Viana MN, Junqueira TS, Ribeiro RCL, Cotta RMM. Conselho de segurança alimentar e nutricional: análise do controle social sobre a política estadual de segurança alimentar e nutricional no Estado do Espírito Santo. Saude Soc. 2011;20(2):470-82. http://dx.doi.org/10.1590/S010412902011000200018.

33. Marques AA, Fernandes MGM, Leite IN, Viana RT, Gonçalves MCR, Carvalho AT. Reflexões de agricultores familiares sobre a dinâmica de fornecimento de seus produtos para a alimentação escolar: o caso de Araripe, Ceará. Saude Soc. 2014;23(4):1329-41. http://dx.doi.org/10.1590/S0104-12902014000400017.

34. Segall-Corrêa AM, Marin-Leon L. A segurança alimentar no Brasil: proposição e usos da escala brasileira de medida da insegurança alimentar de 2003 a 2009. Segur Aliment Nutr. 2009;16(2):1-19. http://dx.doi. org/10.20396/san.v16i2.8634782.

35. Osório MM, Ribeiro MA, Costa EC, Silva SPO, Fernandes CE. Disponibilidade familiar de alimentos na Zona da Mata e Semi-Árido do Nordeste do Brasil. Rev Nutr. 2009;22(3):319-29. http://dx.doi.org/10.1590/ S1415-52732009000300002. 